\title{
The International Atomic Energy Agency International Doubly Labelled Water Database: Aims, Scope and Procedures
}

\author{
John R. Speakman a,b Herman Pontzer ${ }^{c, d} \quad$ Jennifer Rood ${ }^{e}$ Hiroyuki Sagayamaf \\ Dale A. Schoellerg Klaas R. Westerterp ${ }^{\text {h }}$ William W. Wong ${ }^{i}$ Yosuke Yamada $^{j}$ \\ Cornelia Loechlk $^{k}$ Alexia J. Murphy-Alfordk \\ ${ }^{a}$ Institute of Genetics and Developmental Biology, Chinese Academy of Sciences, Beijing, China; ${ }^{b}$ Institute of \\ Biological and Environmental Sciences, University of Aberdeen, Aberdeen, UK; ' ${ }^{\circ}$ Evolutionary Anthropology, Duke

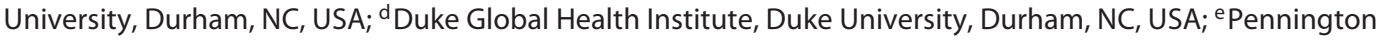 \\ Biomedical Research Center, Baton Rouge, LA, USA; ${ }^{\mathrm{f}}$ Faculty of Health and Sports Sciences, University of Tsukuba, \\ Tsukuba, Japan; ${ }^{9}$ Biotechnology Center and Nutritional Sciences, University of Wisconsin, Madison, WI, USA; \\ ${ }^{h}$ Nutrition and Translational Research in Metabolism, University of Maastricht, Maastricht, The Netherlands; ' USDA/ \\ ARS Children's Nutrition Research Center, Department of Pediatrics, Baylor College of Medicine, Houston, TX, USA; \\ jNational Institute of Health and Nutrition, National Institutes of Biomedical Innovation, Health and Nutrition, \\ Tokyo, Japan; ${ }^{k}$ Division of Human Health, Department of Nuclear Sciences and Applications, International Atomic \\ Energy Agency, Vienna, Austria
}

\section{Keywords}

Energy expenditure - Doubly labelled water - Nutrition . Obesity $\cdot$ Food requirements

\begin{abstract}
Background: The doubly labelled water (DLW) method is an isotope-based technique that quantifies total energy expenditure (TEE) over periods of 1-3 weeks from the differential elimination of stable isotopes of oxygen and hydrogen. The method was invented in the 1950s, but limited ability to measure low isotope enrichments combined with the high cost of isotopes meant it only became feasible to use in humans in the 1980s. It is still relatively expensive to use, and alone small samples are unable to tackle some of the important questions surrounding energy balance such as how have expenditures changed over time and how do expenditures differ with age, between sexes and in different environ-
\end{abstract}

ments? Summary: By combining information across studies, answers to such questions may be possible. The International Atomic Energy Agency (IAEA) DLW database was established to pool DLW data across multiple studies. It was initiated by the main labs currently using the method and is hosted by the IAEA. At present, the database contains 6,621 measures of TEE by DLW from individuals in 23 countries, along with various additional data on the study participants. Key Messages: The IAEA DLW database is a key resource enabling future studies of energy demands.

(C) International Atomic Energy Agency 2019 Published by S. Karger AG, Basel

The article is part of the Proceedings of the International Symposium on Understanding the Double Burden of Malnutrition for Effective Interventions organized by the International Atomic Energy Agency (IAEA) in cooperation with United Nations Children's Fund (UNICEF) and World Health Organization (WHO) (10-13 December 2018, Vienna, Austria).

\begin{tabular}{|c|c|}
\hline KARGER & $\begin{array}{l}\text { (C) International Atomic Energy Agency } 2019 \text { Karger } \\
\text { Published by S. Karger AG, Basel } \\
\text { Open access }\end{array}$ \\
\hline $\begin{array}{l}\text { E-Mail karger@karger.com } \\
\text { www.karger.com/anm }\end{array}$ & $\begin{array}{l}\text { All rights reserved. } \\
\text { This is an Open Access article licensed under the terms of the Creative } \\
\text { Commons Attribution } 3.0 \text { IGO License (CC BY } 3.0 \text { IGO)https://cre- } \\
\text { ativecommons.org/licenses/by/3.0/igo/\#). Usage, distribution and re- } \\
\text { production in any medium or format, even for commercial purposes, } \\
\text { is permitted, provided the original work is properly cited. }\end{array}$ \\
\hline
\end{tabular}

John R. Speakman

Institute of Genetics and Developmental Biology

Chinese Academy of Sciences, Olympic Science park, 1

Beichenxilu, Chaoyang, Beijing 100101 (China)

E Mail J.Speakman@abdn.ac.uk is permitted, provided the original work is properly cited. 


\section{Introduction}

Many key questions in the study of modern nutrition revolve around energy. These include whether the supply of energy is sufficient to meet demands, particularly during the period of growth, and the converse problem of whether supply is too great leading to excess energy intake and obesity and its associated comorbidities. These seemingly contrasting problems often occur side by side and are widely recognized as the double burden of malnutrition. Central to understanding these issues is the need to have accurate measurements of energy demands of free-living individuals. The gold standard method for free-living energy expenditure is the doubly labelled water (DLW) method [1], an isotope-based technique for the assessment of energy expenditure.

The DLW technique is based on the difference between the turnover rates of the hydrogen and oxygen of body water that are a function of carbon dioxide $\left(\mathrm{CO}_{2}\right)$ production. The method allows the measurement of $\mathrm{CO}_{2}$ production over a period when the subject is completely free living. As $\mathrm{CO}_{2}$ production can be directly related to energy expenditure, DLW gives a reflection of free-living energy expenditure. The method involves dosing subjects with water that contains artificially elevated levels of 2 stable isotopic tracers, deuterium $\left({ }^{2} \mathrm{H}\right)$ and oxygen $\left({ }^{18} \mathrm{O}\right)$, hence doubly labelled. These are both naturally occurring stable isotopes that have background levels of around 150 ppm for deuterium and around 2,000 ppm for ${ }^{18} \mathrm{O}$. When individuals drink a dose of DLW, the levels of these isotopes in their bodies rise to 225-260 and 2,150-2,200 ppm, respectively. These levels are not known to produce any toxic reactions or physiological effects, and the method is sufficiently safe that it is routinely used in potentially vulnerable groups like infants, children and pregnant mothers.

The method was invented in the 1950s by Nathan Lifson and colleagues at the University of Minnesota in the USA $[2,3]$ but was only used on small mammals and birds until the early 1980s mostly because of the cost of the isotopes. The first human application was made by Schoeller and van Santen [4], followed closely by several other groups in Texas [5], the UK [6] and the Netherlands [7]. Subsequently, the use of the method has increased enormously. Since 2000 there have been between 94 and 142 papers published annually that have used the DLW method to measure free-living energy requirements based on a literature search of the ISI Web of Science core collection, generated using the search string
(“DLW" OR “DLW" OR "H2O18” OR “field metabolic rate”; Fig. 1).

An example application of the method was the study by Rosetta et al. [8] to measure the energy demands of chronically malnourished lactating females living in Bangladesh. The individuals involved in this study were approximately 12 months post-partum and were all breastfeeding their infants. They had an average BMI of only 17.4 , indicating severe food shortage, and based on recognized cut-offs, $79 \%$ had chronic energy deficiency. Measured daily energy demands were dependent on if the individuals were workers on tea plantations or nonworkers - with the workers expending around 1.6 MJ per day more than non-workers - a difference of about $23 \%$ on their total demands. Surprisingly, however, milk output assessed simultaneously by isotope transfer to their babies did not differ between the 2 groups. These data provide useful information on the energy requirements for successful lactation in communities under food shortage, some of whom are engaged in strenuous activities. Such accurate information can be obtained in no other way.

\section{The IAEA DLW Database}

The International Atomic Energy Agency (IAEA) database had its genesis in 2014 at a DLW training workshop in Tokyo, Japan, following the "Recent Advances in Calorimetry and Measurement of Energy Metabolism: RACMEM" meeting. The idea of compiling together all the published DLW measurements on humans was proposed, and there were several immediately obvious benefits of compiling such a database. First, the technique is still relatively expensive meaning most studies have a sample size of $<30$ individuals. Alone these samples are unable to tackle some of the big questions in nutritional science. For example, how has total energy expenditure (TEE) changed over time? How do TEEs differ between different lifestyles - for example, hunter gatherers, subsistence farming and modern urban living? How do expenditures differ with age, between sexes and in different socio-economic or physical environments? Combining information across studies in a database makes answering such questions possible. The second big advantage is that different studies have used different calculations to generate the estimates of $\mathrm{CO}_{2}$ production and hence TEE. The variation between calculations is not always trivial. Having the raw data underpinning the calculations would allow 
Fig. 1. Publications in the "web of science" core collection database administered by ISI that used DLW (see text for full search terms). In total, there were 3,185 studies. Use of the method has stabilized at about 120 papers per year since 2000 .

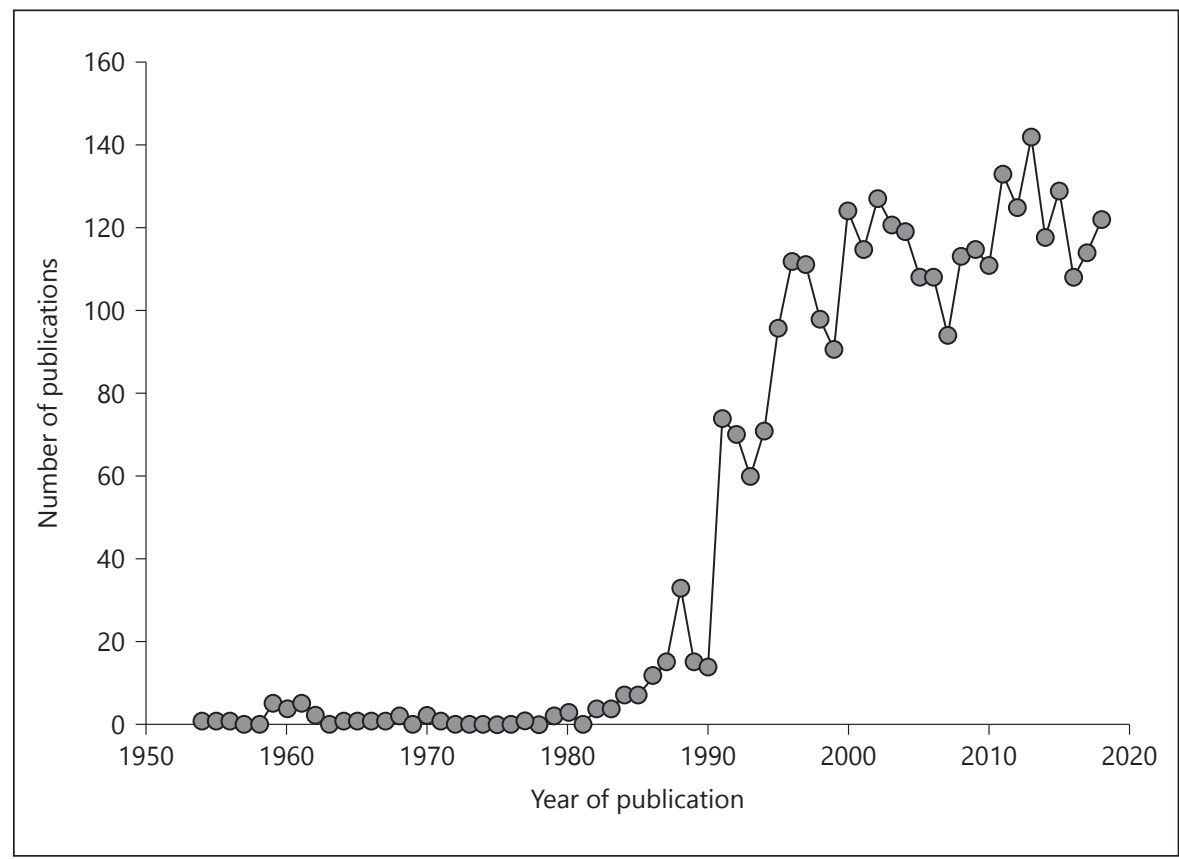

all the data to be recalculated using a single common equation.

The IAEA agreed to host the database, and a total of 6,621 measurements ( 1 record $=1$ measurement on 1 person) have been initially uploaded to the website. The database was formally launched on December 11, 2018 at the International Symposium on the Double Burden of Malnutrition for Effective Interventions, organized by the IAEA in cooperation with WHO and UNICEF in Vienna/Austria. The URL of the website is https://doubly-labelled-water-database.iaea.org/ home.

With over 2,500 published studies applying DLW to humans since the early 1980s, we estimate the number of valid data points that could be included into the database of un-manipulated free-living subjects to be around $20-25,000$. If this estimate is correct, then at present we have captured only about $20 \%$ of the available data. It is the objective of the IAEA database to include as many as possible measurements, and investigators are highly encouraged to submit their records for inclusion, in particular from pre-2000. Individuals submitting data will be acknowledged as authors on any publications that use the database. Additional details regarding data submission is available on the IAEA database website.

If individuals wish to perform an analysis of the data in the database, they should examine the list of currently planned analyses that is available on the website under the "data analysis" tab. A short description of the types of data available in the database can be found on the website under the "data overview tab". The site is interactive and contains the number and type of measurements made in any target country. The detailed steps to apply for performing additional data analysis are described on the IAEA database website.

We would like the findings from all analyses using the database to be published. Manuscripts using the database must adhere to a number of rules that have been agreed by the management group, including draft manuscripts must be approved by the management group, and all contributing authors to the database will be co-authors on papers derived from it. Members of the management group will be co-corresponding authors. This will generate a large authorship list but follows the common practice in many multi-laboratory collaborations. Potential users of the database need to agree to this publication policy before access to perform analyses will be granted. The detailed instructions for potential authors can be viewed on the IAEA database website.

As a flavour of the size of the database and the variability in the data it contains, we show here a simple example analysis; Figure 2 shows the relationship between TEE by DLW (in MJ/day) and body weight ( $\mathrm{kg}$ ) in adults divided by sex. The database includes 3,220 measurements of adult females and 1,810 measurements of adult 

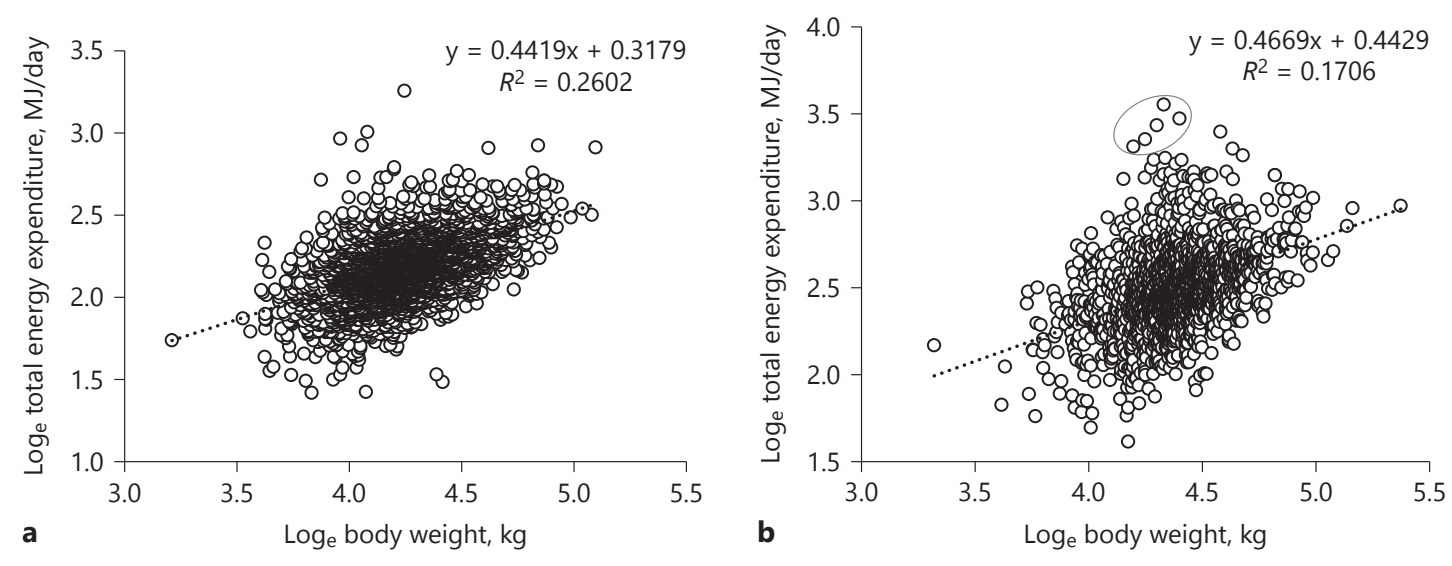

Fig. 2. Total daily energy expenditure (MJ/day) measured by DLW of $(\mathbf{a})$ adult females $(n=3,220)$ and (b) adult males $(n=1,810)$ in relation to body weight $(\mathrm{kg})$. In both cases, heavier people expend-

ed more energy, but there was tremendous individual variation at each body weight. Individuals in the Tour de France cycle race are circled. TEE, total energy expenditure.

males aged between 18 and 96 years. These plots show that energy demands increase directly in relation to body size but that at any chosen weight there is tremendous individual variation. The explained variation in TEE by weight was $26.0 \%$ in females and $17.1 \%$ in males on log transformed data. There was a grade shift in the relationship between males and females. At any given body weight, males expend on average about $1.7 \mathrm{MJ} /$ day more than females. On average, in this sample at the mean age of 54.75 years, the average female expended $9.11 \mathrm{MJ} /$ day (SD 1.8), equivalent to 2,180 kcals per day, and weighed $71.1 \mathrm{~kg}$. In contrast, for the males who in this sample averaged 48.69 years old, the average male expended 12.41 MJ/day (SD 3.2), equivalent to 2,965 $\mathrm{kcal} /$ day and weighed $81.8 \mathrm{~kg}$. These averages are slightly inflated by the inclusion in the males of a small group of athletes engaged in the Tour de France cycle race (circled in Fig. 2b). If these are excluded, the average male expenditure falls to $12.34 \mathrm{MJ} /$ day (equivalent to 2,950 $\mathrm{kcal} /$ day). A key goal of the future analyses of the database is to try and understand the factors that drive the tremendous individual variability that is evident in these plots.

\section{Conclusion}

The DLW method enables unrestricted measures of free-living TEE over periods of 1-3 weeks and is the gold standard method for such measurements. However, the

expense of using it means individual studies generally have small sample sizes. The IAEA-sponsored DLW database aims to combine data from different studies to enable analyses that would not be feasible using individual smaller studies alone. As of May 2019, the database contains 6,621 measurements of TEE.

\section{Acknowledgements}

Susan Roberts kindly hosted a DLW database meeting in Boston in 2015, and Abdul Dulloo hosted one in Fribourg in 2017 for which we are thankful.

\section{Statement of Ethics}

The authors have no ethical conflicts to disclose for this review because there were no humans or animals involved directly.

\section{Disclosure Statement}

The authors have no conflicts of interest to declare.

\section{Funding Sources}

The database is generously supported by the IAEA and by the companies Taiyo Nippon Sanso, SERCON and ISOTEC. We are grateful to these companies for their support and especially to Takashi Oono for his tremendous efforts at fund raising on our be- 
half. The authors also gratefully acknowledge funding from the US National Science Foundation (BCS-1824466) awarded to Herman Pontzer. The funders played no role in the content of this manuscript.

Open access provided with a grant from the International Atomic Energy Agency.

\section{Author Contributions}

All authors contributed to the drafting and editing of the manuscript and to construction of the IAEA DLW database.

\section{Disclaimer}

The statements, opinions and data contained in this publication are solely those of the individual authors and contributors, not of the publishers and the editor(s), and do not necessarily reflect the views of the cooperating organizations, IAEA, UNICEF and WHO. The use of particular designations of countries or territories does not imply any judgement by the cooperating organizations, as to the legal status of such countries or territories, of their authorities and institutions or of the delimitation of their boundaries. The mention of names of specific companies or products (whether or not indicated as registered) does not imply any intention to infringe proprietary rights, nor should it be construed as an endorsement or recommendation on the part of the cooperating organizations.

\section{References}

1 Speakman JR. Doubly-labelled water: Theory and Practice. Springer Berlin; 1997.

2 Lifson N, Gordon GB, Visscher MB, Neir AO. The fate of utilized molecular oxygen and the source of the oxygen of respiratory carbon dioxide, studied with the aid of heavy oxygen. J Biol Chem. 1949 Sep;180(2):80311.

3 Lifson N. Theory of use of the turnover rates of body water for measuring energy and material balance. J Theor Biol. 1966 Sep;12(1):4674.
4 Schoeller DA, van Santen E. Measurement of energy expenditure in humans by doubly labeled water method. J Appl Physiol. 1982 Oct; 53(4):955-9.

5 Klein PD, James WP, Wong WW, Irving CS, Murgatroyd PR, Cabrera M, et al. Calorimetric validation of the doubly-labelled water method for determination of energy expenditure in man. Hum Nutr Clin Nutr. 1984 Mar; 38(2):95-106.

6 Coward WA, Prentice AM. Isotope method for the measurement of carbon dioxide pro- duction rate in man. Am J Clin Nutr. 1985 Mar;41(3):659-63.

7 Westerterp KR, Saris WH, van Es M, ten Hoor F. Use of the doubly labeled water technique in humans during heavy sustained exercise. J Appl Physiol (1985). 1986 Dec;61(6):2162-7.

8 Rosetta L, Kurpad A, Mascie-Taylor N, Shetty PS. Total energy expenditure ((H20)-O18), physical activity level and milk output of lactating rural Bangladeshi tea workers and nontea workers. Eur J Clin Nutr. 2005 Jun;59(5): $632-8$. 